\title{
The Role of Maxillofacial Surgeon in Treatment of Patients with Combined Trauma. Algorithm for a Complex Diagnostic Evaluation
}

\author{
E. Deliverska \\ Department of Oral and Maxillofacial Surgery, Faculty of Dental Medicine, Medical University- Sofia
}

\begin{abstract}
Background: Trauma is a multisystem disease and careful, methodical and duly treatment may optimize the treatment outcome. This requires as quick as possible consultation with a maxillofacial surgeon(MFS), as well as of close multidisciplinary collaboration with other specialists and mainly by neurosurgeon and anaesthesiologist. Purpose: The aim of our study is to define the maxillofacial surgeon role in treatment of combined trauma patients and to suggest an algorithm for a complex diagnostic evaluation. Material and Methods: A total of 352 traumatic patients were retrospectively and prospectively examined for the period 05.2005 12.2011, treated at the MFS Ward at the St. Anna University Multidiscipline Active Treatment Hospital in Sofia, whereas CMFT were determined in 129 patients. Results:During clinical material analysis in our study, it was determined that in combined maxillofacial trauma (CMFT) patients most often consultations were performed with a neurosurgeon - in 109 of examined patients, 21- with traumatologist, 18- with ophthalmologist, 11- with general surgeon, 3- with otorynolaryngologist(ENT). Conclusion:Maxillofacial trauma treatment plays a major part in treatment of polytrauma patients and the importance of early inclusion of maxillofacial surgeon in these patients status estimation and treatment must be emphasized.
\end{abstract}

Keywords: maxillofacial trauma, associated injuries, combined trauma, diagnosis

\section{Introduction}

Trauma may be examined as a multisystem disease and its duly treatment may improve prognosis for the patient. A MFT that is not recognized in due time, often leads to cosmetic and functional disturbances and deformations. The challenge nowadays is to restore the pre-trauma patient's status, but that is not always possible. Continuous development and advancing the treatment methods (directed mainly to tissue engineering and biomechanics) are prerequisites for achieving of excellent results. Maxillofacial surgeon must perform an integral role in interdisciplinary traumatic patients treatment. CMFT require an early multidisciplinary approach because of maxillofacial area unicity, determined by proximity of multiple crucial anatomic structures. The advantage for existence of such team of specialists is in creating of coordinated protocols in combined work, as well as in more arranged and logical approach in these patients treatment. In multiple injuries and severe maxillofacial trauma patients, there is a clinical dilemma if attention to be paid to general status of the patient, thus improving their chance to survive, and prevention of secondary complications is made, or to emphasize on maxillofacial trauma and thus to achieve an excellent result regarding post treatment function and aesthetics. The optimal time for surgical intervention is still under discussion and we consider it is necessary to specify this time depending on particular clinical case.

Understanding the trauma reasons, severity and age structure may support clinical priorities determination, increase treatment effectiveness, and also may achieve certain trauma prevention.

Defining the oral and maxillofacial surgeon role at the emergency centre is closely related to the level of competence and depends on geopolitical and economic factors, on level of education, on knowledge and experience of treatment team. Knowledge and surgical experience, as well as practical skills of oral and maxillofacial surgeon are one of the most important factors in treatment of head and neck trauma patients.

Participation of oral and maxillofacial surgeon is of extreme importance in evaluation of status, in performing diagnostics and in planning of treatment of CMFT patients at the emergency centre (ward). The importance of early maxillofacial surgeon inclusion in status estimation and treatment of polytrauma patients especially must be emphasized.

High head trauma CMFT incidence (which is confirmed by our study as well) supposes mainly a close cooperation between neurosurgeons and maxillofacial surgeons.

In general, oral and maxillofacial surgeon role at the emergency centre is to make $(24,25,26)$ :

a) an assessment of respiratory pathways passability;

b) evaluation of hypovolemia because of maxillofacial region bleeding;

c) evaluation of vision status and head trauma.

During or after the "golden hour", oral and maxillofacial surgeon may perform only consulting activity or various manipulations at the emergency centre. Definitive treatment depends on a series of factors - overall prognosis for trauma outcome, general status of the patient as well as on other planned procedures.

We present a behaviour algorithm in combined traumas and summarize the oral and maxillofacial surgeon role at the emergency centre based on our study and clinical experience:

1) Resuscitation per ATLS - A, B, C, D, E; cervical spine immobilization, vital functions evaluation; estimation of 


\section{International Journal of Science and Research (IJSR) \\ ISSN (Online): 2319-7064}

Index Copernicus Value (2013): 6.14 | Impact Factor (2014): 5.611

need of intubation/tracheostoma, providing a intravenious catheter, fluid resustitation, abdominal ultrasound examination and radiography of thorax.

The maxillofacial and oral surgeon task is in performing control of bleeding/haemostasis and temporary immobilization (when there are no contraindications).

In steady vital functions, various diagnostic procedures are performed.

The maxillofacial and oral surgeon prescribes an imaging diagnostics - radiographies, CT, MRI, ultrasound examination.

In stabilized patients, next comes further diagnostics specification.

The tasks of oral and maxillofacial surgeon are:

a) Administering of $0.5 \mathrm{ml}$ tetanus toxoid i.m.

b) Appointing of consultations with other specialists, if necessary - neurosurgeon, ophthalmologist, traumatologist, general surgeon, ENT. The oral and MF surgeon task is careful and detailed MFA examination, as well as status estimation of the other affected anatomical areas - „A CRASH PLAN". Often, MFS is the one that after a detailed anamnesis and thorough examination may find an omitted combined trauma and to appoint the relevant consultations with other specialists at the emergency ward (sometimes patients are sent directly to the MFS, not passing through the emergency centre and in these cases, detailed examination is extremely important for determining an omitted combined trauma).

Patients with suspected or determined maxillofacial fractures may turn directly to MFS for a treatment. Under these circumstances, injuries of other anatomical body parts may be omitted and therefore it is necessary to make a quick and adequate consultations with the respective specialists.

2) Additional diagnosing, organizing, planning and performing of surgical intervention; preparing of hospital documents by the maxillofacial surgeon and oral surgeon.

Trauma is a multisystem disease and careful, methodical and duly treatment may optimize the treatment outcome. This requires as quick as possible consultation with a MFS, as well as of close multidisciplinary collaboration with other specialists and mainly by neurosurgeon and anaesthesiologist. MFT plays a major part in treatment of polytrauma patients and the importance of early inclusion of maxillofacial surgeon in these patients status estimation and treatment must be emphasized.

The other aim of our study is to suggest an algorithm for a complex diagnostic evaluation

Diagnostic evaluation in patient with maxillofacial trauma (MFT) and associated injuries

Correct diagnostic evaluation in presence of many injuries requires coordinate cooperation of various specialists anaesthesiologist, surgeon, traumatologist, neurosurgeon, ophthalmologist and MFS. Surgeons must coordinate treatment of various types of injuries, whereas thus the combined trauma may be treated precisely and entirely.
During the initial estimation of traumatic patient, systemic approach must be directed to identifying of life threatening and concomitant injuries and subsequently, if any, these to be treated in due time. Regarding the maxillofacial region, clinical examination must be well organized and should better be performed before the imaging diagnostics. Clinical examination is performed in details so that no injury is omitted, and sometimes may be disturbed because of oedemas and suffusions, which could lead to inaccurate evaluation of injury severity and of belonging bone structures.

Disturbances in diagnostics do not only refer to patients with severe MFT. Patients with relatively small MFA injuries may also present a problem during clinical evaluation following a poor cooperation by the patient, vomiting or in case the patient is intoxicated.

In addition, when taking a detailed anamnesis and performing of clinical examination, special attention must be paid to patient status before trauma - occlusion, vision, neurology status.

For MFT treatment, dental models can be very useful to determine the patient's occlusion before the trauma and for preparation of laboratory splints that to help repositioning the fragments.

Diagnostic evaluation is performed on grounds of:

1. Type and classification of trauma

2. Evaluation of trauma severity

2.1. ISS (Injury Severity Score) or AIS (Abbreviated Injury Score).

2.2. In head trauma - evaluation per GCS.

- Light - 15-14 GCS.

- Medial - 13-9 GCS.

- Severe - 8-3 GCS.

3. Trauma mechanism - high energy traumas - falling from a height, RTA, thrash.

4. Trauma morphology - localized or diffuse injuries

5. Evaluation of time factor - primary and secondary injuries

6. Evaluation of patient status - GCS, loss of consciousness, amnesia, neurology deficiency, haemorrhagic diatheses (including anticoagulants administration), age, alcohol intoxication (use of narcotics), headache, vomiting, sudden change in behaviour, evaluation of pupil size, examination of pupil reaction, careful bulb palpation.

7. Imaging diagnostics examination.(16)

Multidisciplinary approach is essential for diagnostics and treatment of combined maxillofacial trauma(CMFT) patients. In order that no omissions in diagnostics are allowed and to achieve optimal patient treatment, the exact duly performing of consultations is required with various adjoining disciplines specialities.

During clinical material analysis in our study, it was determined that in CMFT patients (Table 1,2) most often consultations were performed with a neurosurgeon - in 109 of examined patients, whereas of these:

- independent were in 84 patients;

- combined with an ophthalmologist in 7 patients; 


\section{International Journal of Science and Research (IJSR) \\ ISSN (Online): 2319-7064}

Index Copernicus Value (2013): 6.14 | Impact Factor (2014): 5.611

- combined with a traumatologist in 7 patients;

- combined with an abdominal surgeon in 4 patients;

- combined with a traumatologist and an abdominal surgeon at the same time at 4 patients;

- combined with an ophthalmologist and a traumatologist in 1 patient;

- combined with an ophthalmologist and an abdominal surgeon in 1 patient;

- combined with an abdominal surgeon and ENT in 1 patient.

After the neurosurgeon consultations, most often consultations were performed with an orthopaedisttraumatologist - in 21 patients, of which:

- independent in 9 patients;

- combined with a neurosurgeon in 7 patients;

- combined with a neurosurgeon and an abdominal surgeon in 4 patients;

- combined with a neurosurgeon and an ophthalmologist in 1 patient.

In 18 patients, consultations were performed with an ophthalmologist, of which :

- independent in 8 patients;

- combined with a neurosurgeon in 7 patients;

- combined with ENT in 1 patients;

- combined with a neurosurgeon and orthopaedisttraumatologist in 1 patient;

- combined with a neurosurgeon and an abdominal surgeon in 1 patient.
Consultations with an abdominal surgeon were 11 , of which:

- independent - 1;

- combined with a neurosurgeon - 4;

- combined with a neurosurgeon and a traumatologist - 4;

- combined with a neurosurgeon and an ophthalmologist 1

- combined with a neurosurgeon and ENT - 1.

Consultations with ENT were 3, of which:

- independent - 1;

- combined with an ophthalmologist - 1;

- combined with a neurosurgeon and an abdominal surgeon -1 .

Table 1: Performed consultations with specialists in 129 CMFT patients

\begin{tabular}{|c|c|c|}
\hline \multirow{2}{*}{ Specialist } & \multicolumn{2}{|c|}{ Patients } \\
\cline { 2 - 3 } & Number & $\% *$ \\
\hline Neurosurgeon & 109 & 84.5 \\
\hline Ophthalmologist & 18 & 14 \\
\hline Traumatologist & 21 & 16.3 \\
\hline Abdominal surgeon & 11 & 8.5 \\
\hline ENT/otorhynolaryngologyst & 3 & 2.3 \\
\hline
\end{tabular}

Legend: *Percentage is more than 100 , as in some part of patients, consultations were performed with more than one specialist.

Table 2: CMFT patients traumas and performed consultations with specialists

\begin{tabular}{|l|c|c|c|c|c|c|c|c|c|c|c|c|c|c|}
\hline \multirow{2}{*}{\multicolumn{1}{c|}{ Trauma }} & \multicolumn{9}{|c|}{ Consultations with specialists } & Total \\
\cline { 2 - 13 } & $\mathbf{1}$ & $\mathbf{2}$ & $\mathbf{3}$ & $\mathbf{4}$ & $\mathbf{5}$ & $\mathbf{1 , 2}$ & $\mathbf{1 , 2 , 3}$ & $\mathbf{1 , 2 , 4}$ & $\mathbf{1 , 3}$ & $\mathbf{1 , 3 , 4}$ & $\mathbf{1 , 4}$ & $\mathbf{1 , 4 , 5}$ & $\mathbf{2 , 5}$ & number \\
\hline Neurosurgical & 79 & 1 & 1 & - & - & 3 & 1 & 1 & 1 & 1 & 3 & 1 & - & 92 \\
\hline Of MSS & 1 & - & 8 & - & - & - & - & - & 3 & - & - & - & - & 12 \\
\hline Eye trauma & - & 6 & - & - & - & 3 & - & - & - & - & - & - & 1 & 10 \\
\hline Spinal column & 1 & - & - & - & - & - & - & - & - & 1 & - & - & - & 2 \\
\hline More than 1 CT & - & 1 & - & - & - & 1 & - & - & 2 & - & - & - & - & 4 \\
\hline Polytrauma & 3 & - & - & - & - & - & - & - & 1 & 2 & 1 & - & - & 7 \\
\hline ENT & - & - & - & - & 1 & - & - & - & - & - & - & - & - & 1 \\
\hline Abdominal & - & - & - & 1 & - & - & - & - & - & - & - & - & - & 1 \\
\hline Total number & $\mathbf{8 4}$ & $\mathbf{8}$ & $\mathbf{9}$ & $\mathbf{1}$ & $\mathbf{1}$ & $\mathbf{7}$ & $\mathbf{1}$ & $\mathbf{1}$ & $\mathbf{7}$ & $\mathbf{4}$ & $\mathbf{4}$ & $\mathbf{1}$ & $\mathbf{1}$ & $\mathbf{1 2 9}$ \\
\hline
\end{tabular}

Legend: 1 - a neurosurgeon; 2 - an ophthalmologist; 3 - a traumatologist; 4 - an abdominal surgeon; 5 - ENT

In our study, regarding imaging diagnostics, radiography prevails significantly as a basic imaging diagnostics method in CMFT (in $60.5 \%$ of cases) $(x 2, p<0.001)$. In one third of patients, together with radiography, a computed tomography (CT) examination was used as well. As an independent method, CT is used in only $7.8 \%$ of cases (Table 3 ).

Table 3: Imaging diagnostics methods in CMFT ( $\mathrm{n}=129)$

\begin{tabular}{|l|c|c|c|}
\hline \multirow{2}{*}{ Imaging diagnostics methods } & \multicolumn{2}{|c|}{ Patients } & Level of \\
\cline { 2 - 3 } & Number & $\%$ & significance \\
\hline Radiography & 78 & 60.5 & \multirow{2}{*}{$\mathrm{p}<0.001$} \\
\hline CT & 10 & 7.8 & \multirow{2}{*}{ Radiography + CT } \\
\hline
\end{tabular}

In a part of patients, repeated CT or radiography examination was required, as CT was performed only of cerebral structures, not taking into account possible presence of facial trauma or adequate duly consultation with 


\section{International Journal of Science and Research (IJSR) \\ ISSN (Online): 2319-7064}

Index Copernicus Value (2013): 6.14 | Impact Factor (2014): 5.611

adjoining disciplines specialists have not been performed (Table 4, Figure 1). A CT examination is routine in mid-face trauma patients and is used more and more in diagnostics and evaluation of paediatric traumas. In our study, a CT examination of facial skeleton was performed in 10 patients $(7.8 \%)$, in $41(31.8 \%)$ - radiography, and in $78(60.5 \%)-$ CT examination.
Table 4: Imaging diagnostics methods during the years

\begin{tabular}{|c|c|c|c|c|c|c|}
\hline \multirow{3}{*}{ Year } & \multicolumn{6}{|c|}{ Imaging diagnostics } \\
\cline { 2 - 7 } & Radiography & \multicolumn{2}{c|}{ CT } & \multicolumn{2}{c|}{ Radiography + CT } \\
\cline { 2 - 7 } & Number & $\%$ & Number & $\%$ & Number & $\%$ \\
\hline 2005 & 8 & 61.5 & - & - & 5 & 38.5 \\
\hline 2006 & 15 & 65.2 & 2 & 8.7 & 6 & 26.1 \\
\hline 2007 & 16 & 66.7 & 1 & 4.2 & 7 & 29.2 \\
\hline 2008 & 12 & 57.1 & 3 & 14.3 & 6 & 28.6 \\
\hline 2009 & 6 & 37.5 & 1 & 6.3 & 9 & 56.3 \\
\hline 2010 & 13 & 76.5 & - & - & 4 & 23.5 \\
\hline 2011 & 8 & 53.3 & 3 & 20 & 4 & 26.7 \\
\hline
\end{tabular}

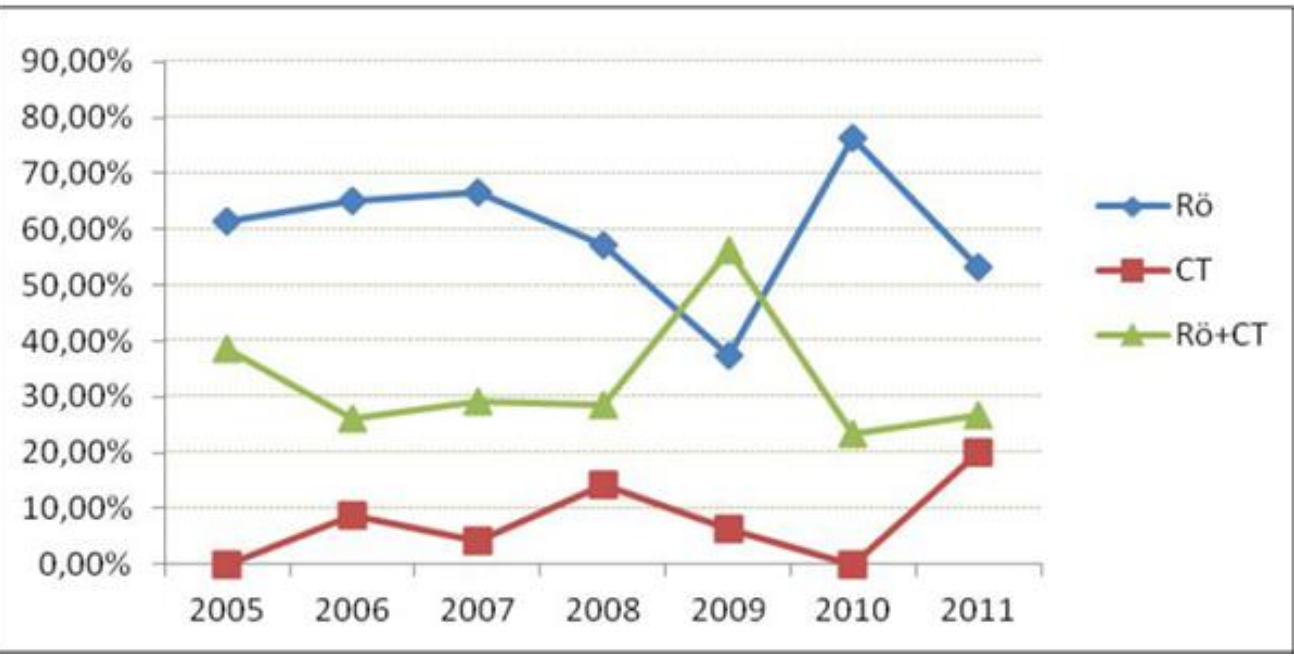

Figure 1: Imaging diagnostics methods during the years

\section{Discussion}

Thoren [30] emphasizes on the fact that MFS must be aware that in $25 \%$ pf MFF there is a concomitant injury, and in our study it is $36,6 \%$.

All specialists must be included in trauma treatment, whereas it is necessary to have the required knowledge and experience in trauma management (Advanced Trauma Life Support), so that no omission in diagnostics and treatment of these patients are allowed. $(1,2,13,14)$

In other study, Down et al. [9] have determined some omissions in trauma diagnostics: from head traumas, omissions are mainly in haematoma diagnostics intracerebral, subdural and epidural, as well as in skull fractures. Omission from the part of MFT: fractures of upper jaw Le Fort II, mandible fracture (body and condyle), teeth trauma are omitted. In abdominal trauma, liver laceration and spleen laceration are omitted; in thoracic trauma, pneumotorax, haemopneumothorax, lung contusion, sternum trauma, rib fracture, myocardial contusion; from MSS traumas, the following were not diagnosed: pelvic fracture, tibia fracture, metatarsal, metacarpal fracture, spinal column injuries were omitted - of cervical and thoracic part.

Bryan Bell et al. [6] publish interesting data in study performed by them. For the period from 1995 to 2005, 20 557 patients have passed through the trauma centre, whereas $17 \%$ of them have had serious MFT and have required MFS consultation. According to consultations with MFS performed, these are on a third place after these with traumatologists and neurosurgeons. From the consultations performed with MFS in the traumatology centre, $42 \%$ were for MFA fractures, 39\% - for soft tissue injuries, 5\% - for tracheostomia, 3\% - for penetrating cervical injury, 3\% - for status evaluation of upper respiratory and digestive systems parts (UADT). The MFT determined by them are: nasal bones fractures - $23 \%$, of orbit - $19 \%$, of zygomatic bone $14 \%$, dentoalveolar $-4 \%$, of frontal sinus - $4 \%$, of maxillary NOS - $13 \%$, facial NOS - $2 \%$, mandibular - $13 \%$, Le Fort I $2 \%$, Le Fort II- $2 \%$, Le Fort III - 4\%. As a leading trauma mechanisms, they indicate RTA - $44 \%$, followed by falling $22 \%$, thrash $-9 \%$, industrial accidents $-7 \%$, sport accidents $9 \%$, other accidents $-3 \%$, firearm accidents - $6 \%$ [6].

Consultations required by MFS after patient's examination, according to the study of Bryan Bell [6] for 2005: with a neurosurgeon - 5 consultations followed by craniotomy; with an ophthalmologist - 72 consultations, with otoneurologist - 30, because of temporal bone fracture, with a laryngologist -6 , in persisting vocal cords paralysis and acording to our dates consultation with neurosurgeon were 109 of examined patients, 21- with traumatologist, 18- with ophthalmologist, 11- with general surgeon, 3- with otorynolaryngologist(ENT).

Imaging diagnostics is often used in evaluation of multiple injuries patients and helps for fast transferring of MFT patients to the MFS wards.

By introducing the new multi slice scanners (high speed with increased number of detectors) [23], a CT examination of MFA allows rapid and precise diagnostics. Examination with these scanners give a high quality image without

\section{Volume 5 Issue 2, February 2016}




\section{International Journal of Science and Research (IJSR) \\ ISSN (Online): 2319-7064 \\ Index Copernicus Value (2013): 6.14 | Impact Factor (2014): 5.611}

artefacts, it is more detailed and rapid, and is connected with less radiation for the patient. Thus, delay in diagnostics and treatment of MFI is avoided [5].

In West Europe countries, there is a trend to reduce the multiple trauma incidence because of reducing heavy industry, improving of motor vehicles safety and developing strict legislative measures against driving under the influence of alcohol [20]. According to bibliographic information, severe trauma is the most common reason for lethal outcome at patients under 40 years of age [20]. A research was conducted in the United Kingdom, according to which 1 on 1000 traumatic patients has had multiple trauma and at the larger hospitals emergency centres, there is at least one such case a week [7]. Medical care expenses, economic activity losses both from financial and from social point of view, are assessed to $102880 £$ per case (Valuation of Road Accidents. Highway Economics Note No. 1, Department of Transport, 1996) [7].

Maxillofacial surgeon (MFS) plays an extremely important and responsible role in diagnostics and treatment planning of CMFT patients. High incience of CMFT with head traumas supposes close cooperation between neurosurgeons and MFS $[4,8,10,12,17,18,19,20]$. In order to not have omissions in diagnostics and therapy of patients, it would be good to include relevant specialists in trauma treatment, with their knowledge and experience in trauma management (Advanced Trauma Life Support) [9,25,26,27,32].

Study of Down et al. [9] is also interesting, indicating that $15 \%$ of seriously traumatic patients that have passed through the hospital, have had concomitant MFT, and according to Bryan Bell [53] - 17\%. According to some authors, basically dentoalveolar (DA) injuries prevail among MFT - 50\% [31]. This CMFT incidence is significant, but much lower than other examinations incidence. According to Thoren et al. [30], there are combined MFT at 25.2\%, and between 53\% and $99.3 \%$ in multiple fracture patients in MFA in other studies [85, 88]. Kruger \& Schilli [21] inform about incidence in $22 \%$ of combined maxillofacial and cerebral trauma (CrT) patients, which is approximately the same in other studies as well (Paschke and Berz, 1961), Muller (1969), Deutchlander-Wolff at al. (1976). Only Van Hoof et al. (1977) [21] indicate higher incidence - every second MFF patient has a head trauma. Muller (1969) [21] indicates that $6.9 \%$ of MFT patients has had fractures on other body parts, while according to Van Hoof et al. [per 124], this percentage is 16.9. Gassner [11] indicates incidence 2:1 to the advantage of male patients, and according to Down et al. [9], incidence is $3: 1$. Thoren [30] also emphasizes on higher incidence of CMFT in male patients - 79.3\%.

Gwyn et al. [15] note that $19 \%$ of hospitalized CMFT patients have had life threatening injuries. Tracheotomy was necessary in $2.3 \%, 1.4 \%$ had lung injury. Fractures on other body parts - in $20 \%$, and $3.4 \%$ had eye injury, which lead to loss of vision of the affected eye. Basing on retrospective and prospective study of Tung et al. [31], life threatening injuries were determined in $6 \%$ of MFT patients, whereas leading injury was cerebral trauma, massive haemorrhage and aeriferous passages damage. Mortality in the study of Tung et al. [31] indicated low values $(0.5 \%)$, and as per
Thoren et al. data - 0.2\% [30]. According to Down et al. [9], about $50 \%$ of MFT patients leave at the trauma place or soon after there have been transported to the hospital. Most such patients never reach MFS and thus the real mortality incidence remains insufficiently estimated in most studies. Some authors indicate that the most frequent life threatening injury is brain trauma [22,28,29,31]. Interesting data about intracranial haemorrhage, determined in the examined MFF patients, are published by Hohlrieder et al. - 9.7\% [19], and Brasileiro \& Passeri $-10.5 \%$ of patients [3].

As a reason for lethal outcome on the place of accident, Down et al. [9] indicate, on the first place, combined trauma - $60 \%$; head trauma - $26 \%$; firearm injuries - $8 \%$; respiratory obstructions - $2 \%$; thorax injuries - $2 \%$; spinal injuries - $2 \%$. While reasons for lethal outcome at hospital are different: head trauma - 64\%, multiple injuries - $15 \%$, spinal injuries $9 \%$, abdominal trauma - 3\%, thorax injuries - 3\%, respiratory obstructions - 3\% [9].

\section{Conclusion}

Severity and complexity of combined maxillofacial traumas require not only multidisciplinary approach, but prevention of these trauma is also of extreme importance, as this may reduce direct and indirect social economic losses.

Development of molecular biology, genetics, resuscitation, intensive treatment during the last years, introducing of new medicines, better understanding of systemic immune response, as well as protocol development and applications in treatment of traumatic patients, provide successful treatment and mortality decrease.

Advanced Trauma Life Support is determined as a golden standard and is based on well known principles, but strict adherence to the protocol may have its disadvantage in presence of a combined MFT. Difficulties in treatment of these patients may arise in presence of a small or a large MFT and both oral surgeon and MFS must be aware that possible problems may appear, regardless of injury severity.

\section{References}

[1] Alvi, A., T. Doherty, G. Lewen. Facial fractures and concomitant injuries in trauma patients. Laryngoscope, 2003, 113: 102-106.

[2] Ardekian, L., D. Rosen, Y. Klein, M. Peled, M. Michaelson, D. Laufer. Life threatening complications and irreversible damage following maxillofacial trauma. Injury, 1998, 29: 253-256.

[3] Brasileiro, B. F., L. A. Passeri: Epidemiological analysis of maxillofacial fractures in Brazil: A 5-year prospective study. Oral Surg. Oral Med. Oral Pathol. Oral Radiol. Endod., 2006, 102: 28.

[4] Brooks, A., B. Holroyd, B. Riley. Missed injury in major trauma patients. Injury, 2004 Apr., 35: 407-410.

[5] Broumand, S. R., J. D. Labs, R. A. Novelline et al. The role of threedimensional computed tomography in the evaluation of acute craniofacial trauma. Ann. Plast. Surg., 1993, 31, 488-494. 


\section{International Journal of Science and Research (IJSR) \\ ISSN (Online): 2319-7064}

Index Copernicus Value (2013): 6.14 | Impact Factor (2014): 5.611

[6] Bryan, Bell, R. The Role of Oral and Maxillofacial Surgery in the Trauma Care Center J. Oral Maxillofac. Surg., 2007, 65: 2544-2553.

[7] Burdett-Smith, P., G. M. Airey, A. J. Franks. Improvement in trauma survival in Leeds. Injury, 1995, 26 (7): 455-458.

[8] Cannell, H, P. V. Dyer, A. Paterson. Maxillofacial injuries in the multiply injured. Eur. J. Emerg. Med., 1996 Mar., 3: 43-47.

[9] Down, K. E., D. A. Boot, D. F. Gorman: Maxillofacial and associated injuries in severely traumatized patients: Implications of a regional survey. Int. J. Oral Maxillofac. Surg., 1995, 24: 409-412.

[10]Follmar, K. E., M. DeBruijn, A. Baccarani et al: Concomitant injuries in patients with panfacial fractures. J. Trauma, 2007, 63: 831

[11] Gassner, R., T. Tuli, O. Hachl, A.Rudisch, H Ulmer Cranio-maxillofacial trauma: a 10 year review of 9534 cases with 21067 injuries. J. Cranio-Maxillofacial Surg., 2003, 31, 51-61.

[12] Gebhard, F., M. Huber-Lang. Polytrauma pathophysiology and management principles. Langenbecks Arch. Surgery, 2008, 393 (6): 825-31.

[13] Grover, R. S., O. M Antonyshyn. Care ofmaxillofacial injuries inmultiple trauma, Mini-Symposium: Nonorthopaedic Aspects of Major Trauma. Current Orthopaedics, 2003, 17, 274-286.

[14] Gruss, J. S., P. J. Bubak, M. A. Egbert. Craniofacial fractures: an algorithm to optimise results. Clin. Plast. Surg., 1992, 19: 195-206

[15] Gwyn, P. P., J. H. Carraway, C. E. Horton et al. Facial fracture - associated injuries and complication. Plast. Reconstr. Surg., 1971, 47: 225-230.

[16]Hardt, N., J. Kuttenberger. Craniofacial trauma Diagnosis and management. 2010.

[17] Haug, R. H., J Prather, A. TIndresano. An epidemiologic Survey of facial fractures and concomitant injuries. Journal of Oral and Maxillofacial Surgery, 1990, 48: 926-932

[18] Hayter, J. P., A. J. Ward, E. J. Smith. Maxillofacial trauma in severely injuried patiaents. Br. J. Oral Maxillofacial Surg., 1991, 29, 370-3.

[19] Hohlrieder, M., J. Hinterhoelzl, H. Ulmer et al: Maxillofacial fractures masking traumatic intracranial hemorrhages. Int. J. Oral Maxillofac. Surg., 2004, 33: 389.

[20]Kloss, F., K. Laimer, M. Hohlrieder et al. Traumatic intracranial haemorrhage in conscious patients with facial fractures. A review of 1959 cases. J. Craniomaxillofac. Surg., 2008, 36: 372.

[21] Kruger, E., W. Schilli. Oral and Maxillofacial surgery. Quintessence Publishing Co, 1982, vol. 1, p. 46

[22] Lim, L. H., L. K. Lam, M. H. Moore et al. Associated injuries in facial fractures: review of 839 patients. Br. J. Plast. Surg., 1993, 46: 635-638.

[23] Melton, S. M., J. D. Kerby, D. McGiffin, G. McGwin, J. K. Smith, R. F. Oser, J. M. Cross, S. T. Windham, S. G. Moran, J. Hsia. Rue 3rd LW. The evolution of chest computed tomography for the definitive diagnosis of blunt aortic injury: a single-center experience. J. Trauma, 2004, 56: 243-250.
[24] Perry, M. Maxillofacial trauma. Developments, innovations and controversies Injury. Int. J. Care Injured 2009, 40, 1252-1259.

[25] Perry, M. Advanced Trauma Life Support (ATLS) and facial trauma: can one size fit all? Part 1: Dilemmas in the management of the multiply injured patient with coexisting facial injuries. Int. J. Oral Maxillofac. Surg., 2008; 37:209-214.

[26] Perry, M., A. Dancey, K. Mireskandari, P. Oakley, S. Davies, M. Cameron. Emergency care in facial trauma-a maxillofacial and ophthalmic perspective. Injury, 2005 Aug., 36: 875-896.

[27] Perry, M., C. Morris: Advanced Trauma Life Support (ATLS) and facial trauma: can one size fit all? Pa Part 2: ATLS, maxillofacial injuries and airway management dilemmas Int. J. Oral Maxillofac. Surg., 2008, 37: 309320.

[28] Simpson, P., J. F. Keating. The multiply injured patient. Acute care the foundation years. Elsevier, 2008, 4: 8

[29] Stadel, P. F., W. R. Smith. Closed head injery. In: Bland KI et al.General surgery: Principles and international practice. 2nd. Adition London: Springer, 2009, p. 13142.

[30] Thoren, H., J. Snall, J. Salo, L. Suominen-Taipale, E. Kormi, C. Lindqvist, J. Tornwall. Incidence and Types of Associated Injuries in Patients With Fractures of the Facial Bones 2010. American Association of Oral and Maxillofacial Surgeons. J. Oral Maxillofac. Surg., 2010, 68: 805-810.

[31] Tung, T. C., W. S .Tseng, C. T. Chen, J. P. Lai, Y. R. Chen. Acute life threatening injuries in facial fracture patients: a review of 1025 patients. J.Trauma, 2000, 49: 420-424

[32] Van Olden, G. D., J. D. Meeuwis, H. W. Bolhuis, H. Boxma, R. J. Goris. Clinical impact of advanced trauma life support. Am. J. Emerg. Med., 2004 Nov., 22: 522525. 